\title{
An Iterative Intercarrier-Interference Reduction Algorithm for OFDM Systems
}

\author{
Y. J. Kou, W.-S. Lu, and A. Antoniou \\ Department of Electrical and Computer Engineering, University of Victoria \\ P.O. Box 3055, Victoria, B.C., Canada V8W 3P6 \\ Email: \{ykou,wslu\}@ece.uvic.ca, aantoniou@ieee.org
}

\begin{abstract}
A low-complexity iterative algorithm is proposed for intercarrier-interference reduction in orthogonal frequency-division multiplexing systems. Design examples are presented which demonstrate that the proposed algorithm outperforms several existing algorithms in terms of bit-error-rate performance and computational complexity.
\end{abstract}

\section{INTRODUCTION}

The demand for high data rate services over wireless networks has been increasing very rapidly and there is no slowdown in sight. These services often require reliable data transmission over band-limited wireless channels, which experience many degradations, such as noise, multipath fading and nonlinearities. A physical-layer technique that has gained much popularity due to its robustness in dealing with these impairments is orthogonal frequency-division multiplexing (OFDM). Well known examples of OFDM modulationbased systems include digital audio broadcasting (DAB) [1], digital video broadcasting (DVB) [2], and the IEEE 802.11a and 802.11g standards [3] for wireless local area networks (WLAN).

Unfortunately, there are several drawbacks associated with OFDM modulation [4]. In a rapidly fading environment, channel variations within an OFDM symbol duration lead to a loss of orthogonality in the OFDM subcarrier waveforms and result in intercarrier interference (ICI) which, in turn, degrades the bit-error-rate (BER) performance of the system [5]-[8]. If not compensated for, ICI will result in an error floor that increases with Doppler frequency. However, channel variations also introduce frequency diversity which can be exploited to improve the system performance [9]. Recently, a number of algorithms have been proposed to mitigate the effect of ICI [10]-[12]. An optimal linear pre-filtering algorithm has been developed in [10] where improved performance was achieved at a cost of increasd computational complexity. In [11], a linear minimum mean-square error (MMSE) has been proposed. Since the number of subcarriers is generally quite large, this algorithm requires intensive computation. In attempts to reduce the computational complexity, a decision feedback (DF) algorithm has been derived in [12] where only signals on several neighbouring subcarriers are used in order to suppress the ICI for a particular subcarrier. The computational complexity of this algorithm is reduced at the cost of a slight degradation of performance.

In this paper, a low-complexity ICI-reduction algorithm based on an iterative optimization scheme is proposed for OFDM systems where 4-quadrature-amplitude-modulation (4-QAM) is assumed for all subcarriers. Design examples are presented which demonstrate that the proposed algorithm outperforms several existing algorithms in terms of BER performance and computational complexity. It is also shown that a better performance can be achieved by the proposed algorithm at higher Doppler frequencies because of the frequency diversity introduced by channel variations.

\section{Signal Model}

Consider an $N$-subcarrier OFDM transmitter as illustrated in Fig. 1, where $\mathrm{S} / \mathrm{P}, \mathrm{P} / \mathrm{S}$, and $\mathrm{DAC}$ represent serial-to-parallel, parallel-to-serial, and digital-to-analog converters, respectively, and the block labeled as "Amp." represents a power amplifier (PA). The information bits $D_{k}$ and the modulated symbol $X_{k}$ are referred to as the data point and subsymbol at the $k$ th subcarrier, respectively. Vectors $\mathbf{X}=\left[X_{0}, \ldots, X_{N-1}\right]^{T}$ and $\mathbf{x}=\left[x_{0}, \ldots, x_{N-1}\right]^{T}$ are referred to as the frequency-domain and the time-domain OFDM symbols, respectively.

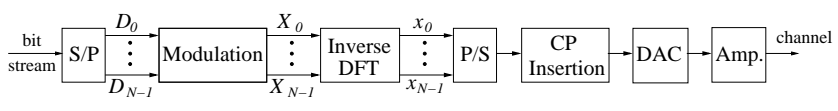

Fig. 1. An OFDM transmitter.

Mathmetically, the OFDM symbol $\mathbf{x}$ can be obtained by using the inverse discrete Fourier transform (IDFT) as

$$
x_{n}=\frac{1}{N} \sum_{k=0}^{N-1} X_{k} e^{j 2 \pi k n / N} \quad \text { for } n=0, \ldots, N-1
$$

where $x_{n}$ represents the $n$th element of $\mathbf{x}$. In matrix form, (1) can be expressed as

$$
\mathbf{x}=\mathbf{Q X}
$$

where $\mathbf{Q}$ is the IDFT matrix whose elements are $q_{n, k}=$ $(1 / N) e^{j 2 \pi k n / N}$. A cyclic prefix (CP) with length equal to that of the channel impulse response (CIR) is inserted in the beginning of the OFDM symbol before it is transmitted into the channel. Denoting the transmitted and received signals as $\mathbf{x}_{C P}=$ $\left[x_{N-L+1}, \ldots, x_{N-1}, x_{0}, \ldots, x_{N-1}\right]^{T}$ and $\mathbf{y}=\left[y_{0}, \ldots, y_{N-1}\right]^{T}$, respectively, the received signal can be written as

$$
\mathbf{y}=\mathbf{H}_{C P} \mathbf{x}_{C P}+\mathbf{n}
$$

where $\mathbf{n}=\left[n_{0}, \ldots, n_{N-1}\right]^{T}$ is a vector of additive white Guassian noise (AWGN) variables with zero mean and covariance matrix $\mathcal{E}\left[\mathbf{n n}^{H}\right]=\sigma^{2} \mathbf{I}$, and the channel matrix $\mathbf{H}_{C P}$ is given by

$\mathbf{H}_{C P}=\left[\begin{array}{cccccccc}h_{0}^{L-1} & h_{0}^{L-2} & \ldots & h_{0}^{0} & 0 & \cdots & 0 & 0 \\ 0 & h_{1}^{L-1} & \cdots & h_{1}^{1} & h_{1}^{0} & \cdots & 0 & 0 \\ \vdots & \vdots & \ddots & \vdots & \vdots & \ddots & \vdots & \vdots \\ 0 & 0 & \cdots & 0 & 0 & \cdots & h_{N-1}^{1} & h_{N-1}^{0}\end{array}\right]$

where $h_{n}^{l}$ for $n=0, \ldots, N-1$ and $l=1, \ldots, l$ represents the fading coefficient of the $l$ th path at the $n$th sample instance. Since the CP is only a copy of part of the OFDM symbol $\mathbf{x}$, (3) can be rewritten as

$$
\mathbf{y}=\mathbf{H x}+\mathbf{n}
$$


where

$$
\mathbf{H}=\left[\begin{array}{ccccc}
h_{0}^{0} & 0 & \cdots & h_{0}^{2} & h_{0}^{1} \\
h_{1}^{1} & h_{1}^{0} & \cdots & h_{1}^{3} & h_{1}^{2} \\
\vdots & \vdots & \ddots & \vdots & \vdots \\
h_{L-1}^{L-1} & h_{L-1}^{L-2} & \cdots & 0 & 0 \\
\vdots & \vdots & \ddots & \vdots & \vdots \\
0 & 0 & \cdots & h_{N-2}^{0} & 0 \\
0 & 0 & \cdots & h_{N-1}^{1} & h_{N-1}^{0}
\end{array}\right]
$$

At the receiver, after the removal of the $\mathrm{CP}$, the received signal is transformed to $\mathbf{Y}=\left[Y_{0}, \ldots, Y_{N-1}\right]^{T}$ by using the discrete Fourier transform (DFT) as

$$
\mathbf{Y}=\mathbf{Q}^{H} \mathbf{y}
$$

where $(\cdot)^{H}$ represents matrix Hermitian. From (2), (5a), and (6), Y can be expressed as

$$
\mathbf{Y}=\mathbf{A X}+\mathbf{N}
$$

where

$$
\mathbf{A}=\mathbf{Q}^{H} \mathbf{H} \mathbf{Q}
$$

and $\mathbf{N}=\left[N_{0}, \ldots, N_{N-1}\right]^{T}=\mathbf{Q}^{H} \mathbf{n}$. Since the DFT matrix $\mathbf{Q}^{H}$ is unitary, $\mathbf{N}$ in (7a) is still white Guassian noise. Due to channel variations, matrix $\mathbf{A}$ is not a diagonal matrix. In particular, the signal at the $k$ th subcarrier can be written as

$$
Y_{k}=A_{k, k} X_{k}+\sum_{i=0, i \neq k}^{N-1} A_{k, i} X_{i}+N_{k}
$$

for $k=0, \ldots, N-1$ where $A_{k, i}$ represents the $(k, i)$ th element of matrix A. It can be seen that for the $k$ th subcarrier the received signal depends not only on the transmitted signal for this particular subcarrier but also on the transmitted signals for other subcarriers. The first and second terms on the right-hand side (r.h.s.) of (8) represent the attenuated signal and the ICI at the $k$ th subcarrier, respectively.

In order to mitigate the effect of the ICI, joint detection (JD) is required at the receiver. This can be done by inserting immediately after the DFT a processor that implements a JD algorithm. A general structure for the OFDM receiver that implements a JD algorithm is illustrated in Fig. 2. For the signal detection problem in (7), maximum likelihood (ML) detection [13] can be carried out by solving the optimization problem

$$
\begin{gathered}
\operatorname{minimize}\|\mathbf{Y}-\mathbf{A X X}\|_{2}^{2} \\
\text { subject to : } X_{k} \in \mathcal{M} \quad \text { for } k=0,1, \ldots, N-1
\end{gathered}
$$

where $\mathcal{M}$ is the set of the constellation points associated with modulation scheme of the OFDM system. The problem in (9) is a combinatorial optimization problem whose solution requires computational complexity that grows exponentially with the number of variables. In addition, in OFDM systems over frequency-selective fading channels, complete information on the CIR is required for ML detection. In what follows, a low-complexity algorithm is proposed that can be used to achieve a suboptimal solution of the optimization problem in (9).

\section{An ITERATIVE ALgORITHM FOR ICI REDUCTION}

The variables in (9) are complex-valued. If we let $\mathbf{Y}=\mathbf{Y}_{r}+j \mathbf{Y}_{i}$, $\mathbf{A}=\mathbf{A}_{r}+j \mathbf{A}_{i}$, and $\mathbf{X}=\mathbf{X}_{r}+j \mathbf{X}_{i}$, then the norm in (9a) assumes the form

$$
\|\tilde{\mathbf{Y}}-\tilde{\mathbf{A}} \tilde{\mathbf{X}}\|_{2}^{2}
$$

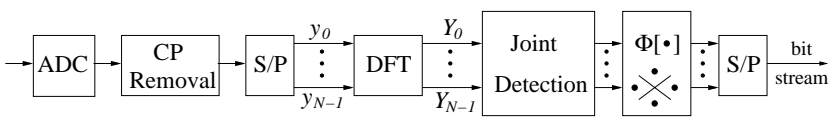

Fig. 2. An OFDM receiver.

where

$$
\tilde{\mathbf{Y}}=\left[\begin{array}{l}
\mathbf{Y}_{r} \\
\mathbf{Y}_{i}
\end{array}\right], \tilde{\mathbf{A}}=\left[\begin{array}{rr}
\mathbf{A}_{r} & -\mathbf{A}_{i} \\
\mathbf{A}_{i} & \mathbf{A}_{r}
\end{array}\right], \tilde{\mathbf{X}}=\left[\begin{array}{l}
\mathbf{X}_{r} \\
\mathbf{X}_{i}
\end{array}\right]
$$

and the problem in (9) can be expressed as

$$
\begin{gathered}
\operatorname{minimize} \quad\|\tilde{\mathbf{Y}}-\tilde{\mathbf{A}} \tilde{\mathbf{X}}\|_{2}^{2} \\
\text { subject to : } \tilde{X}(i) \in \tilde{\mathcal{M}} \quad \text { for } i=0,1, \ldots, 2 N-1
\end{gathered}
$$

where $\tilde{\mathcal{M}}$ represents the set of points associated with the real and imaginary components of the modulation constellation. For 4-QAM modulation, we have $\tilde{\mathcal{M}}=\{1,-1\}$.

For the optimization problem in (11), the elements of vector $\tilde{X}$ can be determined iteratively. Assume that the number and the set of indices of the undetermined elements in vector $\tilde{X}$ during the $k$ th iteration are denoted as $N_{k}$ and $\mathcal{I}_{k}$, respectively. During the first iteration, we have $N_{1}=2 N$ and $\mathcal{I}_{1}=\{0, \ldots, 2 N-1\}$. If we define

$$
\begin{aligned}
& \tilde{\mathbf{X}}_{1}=\tilde{\mathbf{X}}\left(\mathcal{I}_{1}\right), \tilde{\mathbf{A}}_{1}=\tilde{\mathbf{A}}\left(:, \mathcal{I}_{1}\right), \tilde{\mathbf{Y}}_{1}=\tilde{\mathbf{Y}} \\
& \mathbf{B}_{1}=2 \tilde{\mathbf{A}}_{1}^{T} \tilde{\mathbf{A}}_{1}, \quad \mathbf{b}_{1}=-2 \tilde{\mathbf{A}}_{1}^{T} \tilde{\mathbf{Y}}_{1}
\end{aligned}
$$

and let $k=1$, the problem in (11) can be converted to

$$
\begin{gathered}
\operatorname{minimize} \frac{1}{2} \tilde{\mathbf{X}}_{k}^{T} \mathbf{B}_{k} \tilde{\mathbf{X}}_{k}+\tilde{\mathbf{X}}_{k}^{T} \mathbf{b}_{k} \\
\text { subject to : } \tilde{X}_{k}(i) \in\{1,-1\} \quad \text { for } i=0, \ldots, N_{k}-1
\end{gathered}
$$

Since the constraints in (13b) imply that $\tilde{\mathbf{X}}_{k}^{T} \tilde{\mathbf{X}}_{k}=2 N_{k}$, the problem in (13) can be relaxed to

$$
\begin{aligned}
& \operatorname{minimize} \frac{1}{2} \tilde{\mathbf{X}}_{k}^{T} \mathbf{B}_{k} \tilde{\mathbf{X}}_{k}+\tilde{\mathbf{X}}_{k}^{T} \mathbf{b}_{k} \\
& \text { subject to : } \tilde{\mathbf{X}}_{k}^{T} \tilde{\mathbf{X}}_{k}=2 N_{k}
\end{aligned}
$$

Note that matrix $\mathbf{B}_{k}$ is a positive definite matrix and, therefore, it can be diagonalized as

$$
\mathbf{B}_{k}=\mathbf{U}_{k}^{T} \mathbf{S}_{k} \mathbf{U}_{k}
$$

where $\mathbf{S}_{k}$ and $\mathbf{U}_{k}$ are diagonal and orthogonal matrices, respectively. If we let

$$
\begin{aligned}
\mathbf{Y}_{k} & =\mathbf{U}_{k} \tilde{\mathbf{X}}_{k} \\
\tilde{\mathbf{b}}_{k} & =\mathbf{U}_{k} \mathbf{b}_{k}
\end{aligned}
$$

and

$$
\tilde{\mathbf{X}}_{k}=\mathbf{U}_{k}^{T} \mathbf{Y}_{k}
$$

then the problem in (14) can be converted to

$$
\begin{aligned}
& \operatorname{minimize} \frac{1}{2} \mathbf{Y}_{k}^{T} \mathbf{S}_{k} \mathbf{Y}_{k}+\mathbf{Y}_{k}^{T} \tilde{\mathbf{b}}_{k} \\
& \text { subject to : } \mathbf{Y}_{k}^{T} \mathbf{Y}_{k}=2 N_{k}
\end{aligned}
$$

Here, we define the Lagrangian

$$
L\left(\mathbf{Y}_{k}, \lambda_{k}\right)=\frac{1}{2} \mathbf{Y}_{k}^{T} \mathbf{S}_{k} \mathbf{Y}_{k}+\mathbf{Y}_{k}^{T} \tilde{\mathbf{b}}_{k}+\lambda_{k}\left(\mathbf{Y}_{k}^{T} \mathbf{Y}_{k}-2 N_{k}\right)
$$


Using the first-order Karush-Kuhn-Tucker (KKT) conditions, we have

$$
\frac{\partial L\left(\mathbf{Y}_{k}, \lambda_{k}\right)}{\partial \mathbf{Y}_{k}}=\left(\mathbf{S}_{k}+2 \lambda_{k} \mathbf{I}_{k}\right) \mathbf{Y}_{k}+\tilde{\mathbf{b}}=0
$$

It can be seen from (17) and (19) that the solution of the problem in (17) can be obtained by solving the following problem

$$
\begin{aligned}
& \mathbf{Y}_{k}=-\left(\mathbf{S}_{k}+2 \lambda_{k} \mathbf{I}_{k}\right)^{-1} \tilde{\mathbf{b}}_{k} \\
& \text { subject to : } \mathbf{Y}_{k}^{T} \mathbf{Y}_{k}=2 N_{k}
\end{aligned}
$$

On replacing vector $\mathbf{Y}_{k}$ in (20b) using the relationship in (20a), we have

$$
\tilde{\mathbf{b}}_{k}^{T}\left(\mathbf{S}_{k}+2 \lambda_{k} \mathbf{I}_{k}\right)^{-2} \tilde{\mathbf{b}}_{k}=2 N_{k}
$$

where $\mathbf{S}_{k}+2 \lambda_{k} \mathbf{I}_{k}$ is a diagonal matrix. Therefore, Eq. (21) can be further expressed as

$$
\sum_{i=0}^{N_{k}-1} \frac{\tilde{b}_{k}^{2}(i)}{\left[S_{k}(i)+2 \lambda_{k}\right]^{2}}=2 N_{k}
$$

where $\tilde{b}_{k}(i)$ and $S_{k}(i)$ represent the $i$ th element of vector $\tilde{\mathbf{b}}_{k}$ and the main diagonal of matrix $\mathbf{S}_{k}$, respectively. The only unknown variable in (22) is $\lambda_{k}$ and its value can be numerically determined by solving the following one-dimensional optimization problem

$$
\begin{array}{l|l}
\operatorname{minimize} & \left|\sum_{i=0}^{N_{k}-1} \frac{\tilde{b}_{k}^{2}(i)}{\left[S_{k}(i)+2 \lambda_{k}\right]^{2}}-2 N_{k}\right| \\
\text { subject to : } \lambda_{L} \leq \lambda_{k} \leq \lambda_{U}
\end{array}
$$

where

$$
\lambda_{L}=-\frac{1}{2} \min _{0 \leq i \leq N_{k}-1} S_{k}(i)+\epsilon, \lambda_{U}=\frac{1}{2} \max _{0 \leq i \leq N_{k}-1}\left|\tilde{b}_{i}\right|
$$

and $\epsilon$ is a small positive number. The solution of the problem in (23) can be found using, for example, a dichotomous search algorithm. When $\lambda_{k}$ is found, vector $\mathbf{Y}_{k}$ can be computed as

$$
Y_{k}(i)=-\frac{\tilde{b}_{k}(i)}{S_{k}(i)+2 \lambda_{k}} \quad \text { for } i=0, \ldots, N_{k}-1
$$

where $Y_{k}(i)$ is the $i$ th element of vector $\mathbf{Y}_{k}$. Using (16c), an estimzation of the vector $\tilde{\mathbf{X}}_{k}$ can be obtained as

$$
\overline{\mathbf{X}}_{k}=\mathbf{U}_{k}^{T} \mathbf{Y}_{k}
$$

In order to obtain the discrete-valued vector, a decision process is applied where

$$
\hat{X}_{k}(i)= \begin{cases}1 & \text { if } \bar{X}_{k}(i)>\alpha \\ -1 & \text { if } \bar{X}_{k}(i)<-\alpha \\ \text { undetermined } & \text { otherwise }\end{cases}
$$

for $i=0, \ldots, N_{k}-1$ and $\alpha$ is the decision threthold. If $\alpha=0$, then the decision process in (17) becomes the hard decision

$$
\hat{\mathbf{X}}_{k}=\operatorname{sign}\left[\overline{\mathbf{X}}_{k}\right]
$$

and all the elements of vector $\hat{\mathbf{X}}_{k}$ can be determined by using (18). If $\alpha>0$, some of the elements of vector $\overline{\mathbf{X}}_{k}$ may fall into the region where $\left|\bar{X}_{k}(i)\right| \leq \alpha$, and, therefore, these elements cannot be determined in the present iteration. In such a case, more iterations are needed and a feedback scheme can be exploited. Denoting the set of indices of the elements of $\tilde{\mathbf{X}}$ that have been determined in the $k$ th iteration as $\mathcal{I}_{k}^{d}$, we have $\mathcal{I}_{k+1}=\mathcal{I}_{k}-\mathcal{I}_{k}^{d}$ and

$$
\begin{aligned}
& \tilde{\mathbf{X}}_{k+1}=\tilde{\mathbf{X}}\left(\mathcal{I}_{k+1}\right), \tilde{\mathbf{A}}_{k+1}=\tilde{\mathbf{A}}\left(:, \mathcal{I}_{k+1}\right), \\
& \tilde{\mathbf{Y}}_{k+1}=\tilde{\mathbf{Y}}_{k}-\tilde{\mathbf{A}}\left(:, \mathcal{I}_{k}^{d}\right) \tilde{\mathbf{X}}\left(\mathcal{I}_{k}^{d}\right), \mathbf{B}_{k+1}=2 \tilde{\mathbf{A}}_{k+1}^{T} \tilde{\mathbf{A}}_{k+1}, \\
& \mathbf{b}_{k+1}=-2 \tilde{\mathbf{A}}_{k+1}^{T} \tilde{\mathbf{Y}}_{k+1}
\end{aligned}
$$

Based on the updated variables in (28), a similar optimization problem can be formulated as in (13) for the $(k+1)$ th iteration, which can be solved following the same procedure. When all the elements of vector $\tilde{\mathbf{X}}$ are determined, i.e., set $\mathcal{I}_{k+1}$ is empty, the algorithm terminates. A step-by-step description of the above algorithm is listed in Table 1.

TABLE I

\section{AN ITERATIVE OPTIMIZATION ALGORITHM FOR ICI REDUCTION}

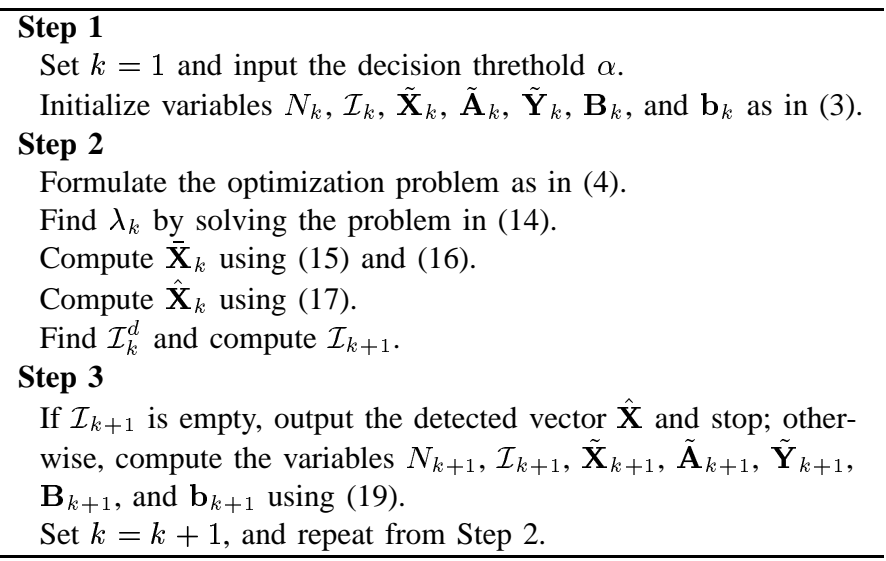

\section{Simulations}

The proposed ICI-reduction algorithm was applied to an OFDM system where the number of subcarriers was chosen to be 64 and 4-QAM was adopted as the modulation scheme for each subcarrier. The bandwidth of the OFDM system and the carrier frequency were set to $B=200 \mathrm{kHz}$ and $f_{c}=2 \mathrm{GHz}$, respectively. The length of the CP was set to $N_{p} T_{c}=(N / 8) T_{c}=T_{s} / 8$, where $T_{s}$ and $T_{c}$ are the time durations of OFDM symbols and chips, respectively. A two-tap Rayleigh fading channel model [12] was assumed where the Doppler frequency of the channel is denoted as $f_{D}$. While the delay of the first tap was zero, the delay of the second tap was randomly generated with uniform distribution from $\left\{T_{c}, 2 T_{c}, \ldots, N_{p} T_{c}\right\}$. The BER performance of the proposed algorithm was evaluated and compared with that of several existing algorithms under a variety of system configurations. For the DF algorithm in [12], the number of neighbouring subcarriers that were used to suppress the ICI at a particular subcarrier was taken to be $2 K+1$.

Example: First we considered an OFDM system where $f_{D} T_{s}=0.05$. For the proposed ICI-reduction algorithm with $\alpha=0.9$ or $a=1.0$, the BER versus the ratio of energy-per-bit to spectral noise density (Eb/N0) is plotted as the solid and dash curves in Fig. 3, respectively. For the sake of comparison, the BER of the DF algorithm in [12] is plotted in the same figure for various values of $K$ as dotted curves. It can be observed that improved performance can be obtained by the proposed algorithm compared with that of the DF algorithm for less computation. For example, at the BER level of $10^{-3}$, a $0.5-\mathrm{dB}$ and 1.5-dB improvement of Eb/N0 can be achieved by the proposed algorithm with $\alpha=0.9$ compared with the DF algorithm with $K=$ 5 and $K=15$, respectively. It was found out that the CPU time 
required by the proposed algorithm with $\alpha=0.9$ is only $80 \%$ of that required by the DF algorithm with $K=15$.

The performance of the proposed algorithm for the cases where $f_{D} T_{s}=0.1$ and $f_{D} T_{s}=0.25$ is plotted in Fig. 4 and 5, respectively. As can be seen, the performance of the algorithm improves with an increase in the Doppler frequency. For example, while for the case of $f_{D} T_{s}=0.1$ an $E b / N 0$ ratio of $27.5 \mathrm{~dB}$ is required to achieve the BER level of $10^{-3}$, for the case of $f_{D} T_{s}=0.25$ an $E b / N 0$ ratio of $25.5 \mathrm{~dB}$ is required to achieve the same BER level. This improvement with increasing $f_{D}$ can be attributed to the increase in frequency diversity introduced by the higher Doppler spread [9].

\section{Conclusions}

A low-complexity ICI-reduction algorithm based on an iterative optimization scheme has been proposed. Design examples have been presented which demonstrate that the proposed algorithm outperforms several existing algorithms in terms of bit-error-rate performance and computational complexity. It has also been shown that the proposed algorithm exploits the frequency diversity introduced by channel variations and, therefore, improved performance can be achieved at higher Doppler frequencies.

\section{ACKNOWLEDGEMENT}

The authors are grateful to Micronet, NCE Program, and the Natural Sciences and Engineering Research Council of Canada for supporting this work.

\section{REFERENCES}

[1] ETSI, "Radio broadcasting systems: digital audio broadcasting to mobile, portable and fixed receivers," European Telecommunication Standard, ETS 300-401, Feb. 1995.

[2] ETSI, "Digital video broadcasting: framing structure, channel coding, and modulation for digital terrestrial television," European Telecommunication Standard, ETS 300-744, Aug. 1997.

[3] IEEE 802.11, "IEEE Standard for Wireless LAN Medium Access Control (MAC) and Physical Layer (PHY) specifications," Nov. 1997.

[4] R. V. Nee and R. Prasad, OFDM for Wireless Multimedia Communications, Artech House Publisher, 2000.

[5] M. Russell and G. L. S. Stuber, "Interchannel interference analysis of OFDM in a mobile environment," Proc. IEEE VTC, vol. 2, pp. 820-824, Jul. 1995.

[6] P. Robertson and S. Kaiser, "The effects of Dopper spreads in OFDM(A) mobile radio systems," Proc. IEEE VTC, vol. 1, pp. 329333, Sep. 1999.

[7] P. Robertson and S. Kaiser, "Analysis of the loss of orthogonality through Doppler spread in OFDM systems," Proc. IEEE GLOBLECOM, vol. 1b, pp. 701-706, Dec. 1999.

[8] Y. Li and L. J. Cimini, Jr., "Bounds on the interchannel interference of OFDM in time-varying impairments," IEEE Trans. Commun., vol. 49, pp. 401-404, Mar. 2001.

[9] N. J. Bass and D. P. Taylor, "Matched filter bounds for wireless communication over Rayleigh fading dispersive channel," IEEE Trans. Commun., vol. 49, pp. 1525-1528, Sep. 2001.

[10] A. Stamoulis, S. N. Diggavi, and N. A. Dhahir, "Intercarrier interference in MIMO OFDM," IEEE Trans. Commun., vol. 50, pp. 2451-2464, Oct. 2002.

[11] Y.-S. Choi, P. J. Voltz, and F. A. Cassara, "On channel estimation and detection for multicarrier signals in fast and selective Rayleigh fading channels," IEEE Trans. Commun., vol. 49, pp. 1375-1387, Aug. 2001.

[12] X. Cai and G. B. Giannakis, "Bounding performance and suppressing intercarrier interference in wireless mobile OFDM," IEEE Trans. Commun., vol. 51, pp. 2047-2056, Dec. 2003.
[13] J. G. Proakis, Digital Communications, 4th ed., McGraw-Hill, 2000.

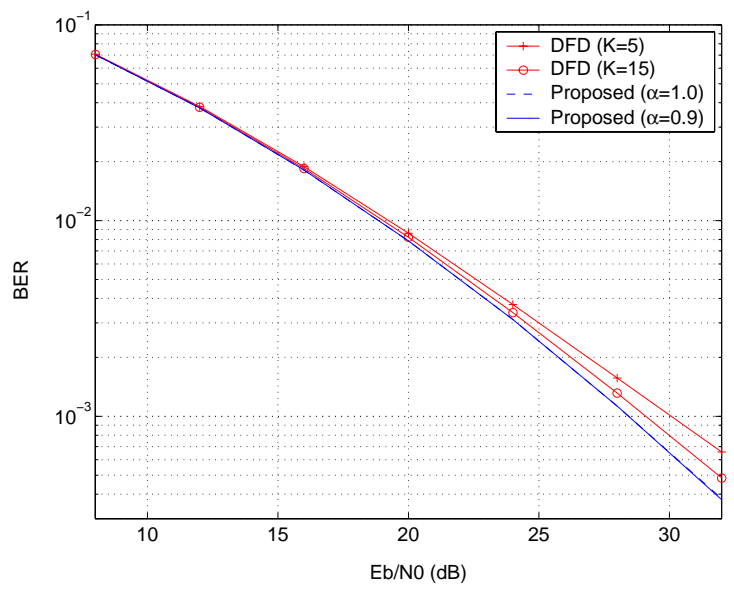

Fig. 3. Performance of ICI-reduction algorithms with $f_{d} T_{s}=0.05$.

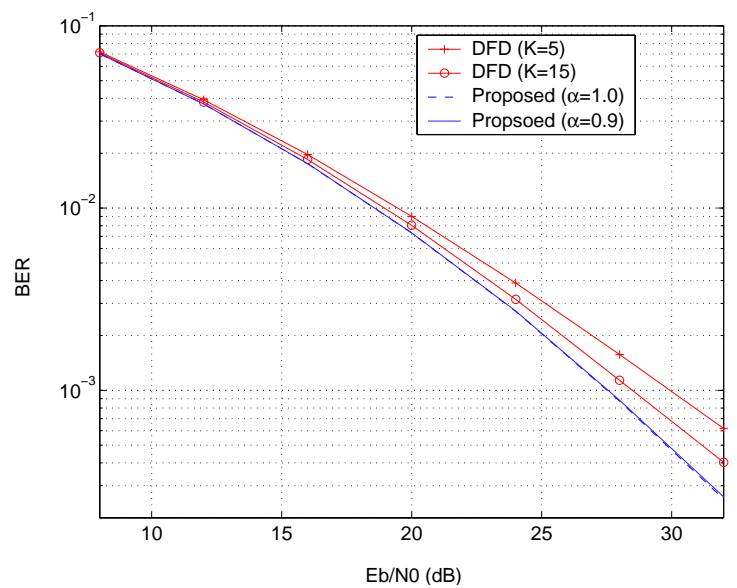

Fig. 4. Performance of ICI-reduction algorithms with $f_{d} T_{s}=0.1$.

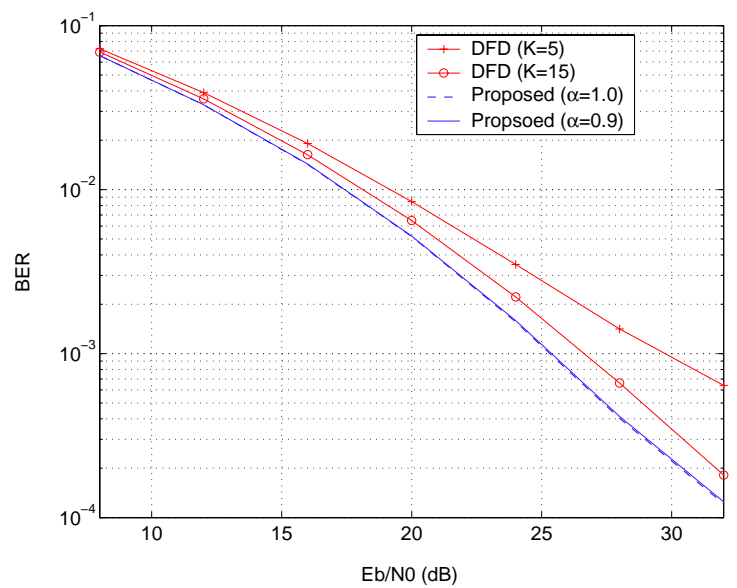

Fig. 5. Performance of ICI-reduction algorithms with $f_{d} T_{s}=0.25$. 